\title{
A Suggestive Case Report of Situs Inversus Totalis of a Young Boy at Computed Tomography
}

\author{
Francesco Messina*, Lorena Turano and Nicola Arcadi \\ Radiology Unit-Riuniti Hospital, Azienda Ospedaliera Grande Ospedale Metropolitano “Bianchi-Melacrino-Morelli", Reggio Calabria, Italy \\ *Corresponding author: Francesco Messina, Radiology Unit-Riuniti Hospital, Azienda Ospedaliera Grande Ospedale Metropolitano \\ "Bianchi-Melacrino-Morelli", Reggio Calabria, Italy.
}

To Cite This Article: Francesco Messina, Lorena Turano, Nicola Arcadi. A Suggestive Case Report of Situs Inversus Totalis of a Young Boy at Computed Tomography. Am J Biomed Sci \& Res. 2021 - 13(3). AJBSR.MS.ID.001869. DOI: 10.34297/AJBSR.2021.13.001869.

Received: 制 May 31, 2021; Published: 制 June 28, 2021

\begin{abstract}
Situs inversus totalis (SIT) is a rare recessive autosomal congenital abnormality in which the mediastinal and abdominal organs are in a mirrored position when compared to the usual topography. We present the case of a 35-year-old male patient with SIT, detected by Computed Tomography (CT), that presented to the Emergency Department with abdominal pain.
\end{abstract}

Keywords: Situs Inversus; Dextrocardia; Computed Tomography; MPR; Mirror-Image

\section{Case Presentation}

A 35-year-old male patient presented at Emergency Department with abdominal pain since about three weeks, mostly in the inferior abdominal quadrants, and at a deep palpation. No fever, and arterial oxygen saturation (SaO2) was 98\%. The naso-pharyngeal sampling for SARS-CoV-2 was negative. Laboratory exams showed only an increase of the values of C-reactive protein and VES. It was decided firstly to perform an abdomen radiography, and immediately after an unenhanced CT. CT had shown (Figure 1) the presence of a clear situation of situs inversus totalis. In fact, the cardiac apex, aortic arch, stomach, and descending colon were right-sided structures. CT identified a right-rotated cardiac image (dextrocardia), with also an evident rotation of the main vascular structures (thoracic aorta and pulmonary artery). Two lung shadings were identified on the right, and three scissures on the left lung. The liver was located in the left upper quadrant, while the spleen was located in the right upper quadrant. The remaining abdominal structures demonstrated a mirror-image location relative to situs solitus. The abdominal aorta was right-sided, the inferior vena cava was leftsided, the pancreatic head and uncinate process were to the left of midline, and the splenic and superior mesenteric artery and vein had a mirror-image positioning. CT also identified the presence of little diverticula of the descending colon (on the right). The patient, after a surgical consulence, was prescribed drug therapy, rest and food attention (diet) for the diverticula; meanwhile, he is carrying out an US and laboratory monitoring of it.

\section{Discussion}

Situs inversus totalis is defined as the complete inversion of the thoracic and abdominal organs, which constitute a mirror image of the normal anatomy. It was first described by Matthew Baillie in the 16 th century and is a rare, congenital condition, with an overall frequency estimated to be approximately at 1/10000 live births. The syndrome has a genetic background, following an autosomal recessive pattern of inheritance. Subjects with the condition are usually asymptomatic, as the structure and function of vital organs are generally unaffected. Situs inversus is the mirror image of situs solitus. Situs inversus can be further subdivided into situs inversus totalis, and situs inversus with levocardia. Situs inversus totalis is characterized by mirror-image location of the heart and viscera relative to situs solitus. The cardiac apex, single spleen, stomach, jejunum, descending colon, and aorta are right-sided structures. The right lung is bilobed with a hyparterial bronchus, and the left lung is trilobed with an eparterial bronchus. The liver, gallbladder, ligament of Treitz, ileum, ascending colon, and inferior vena cava are left-sided structures. Additionally, the normal superior mesenteric artery and superior mesenteric vein relationship is inverted [1-5]. 

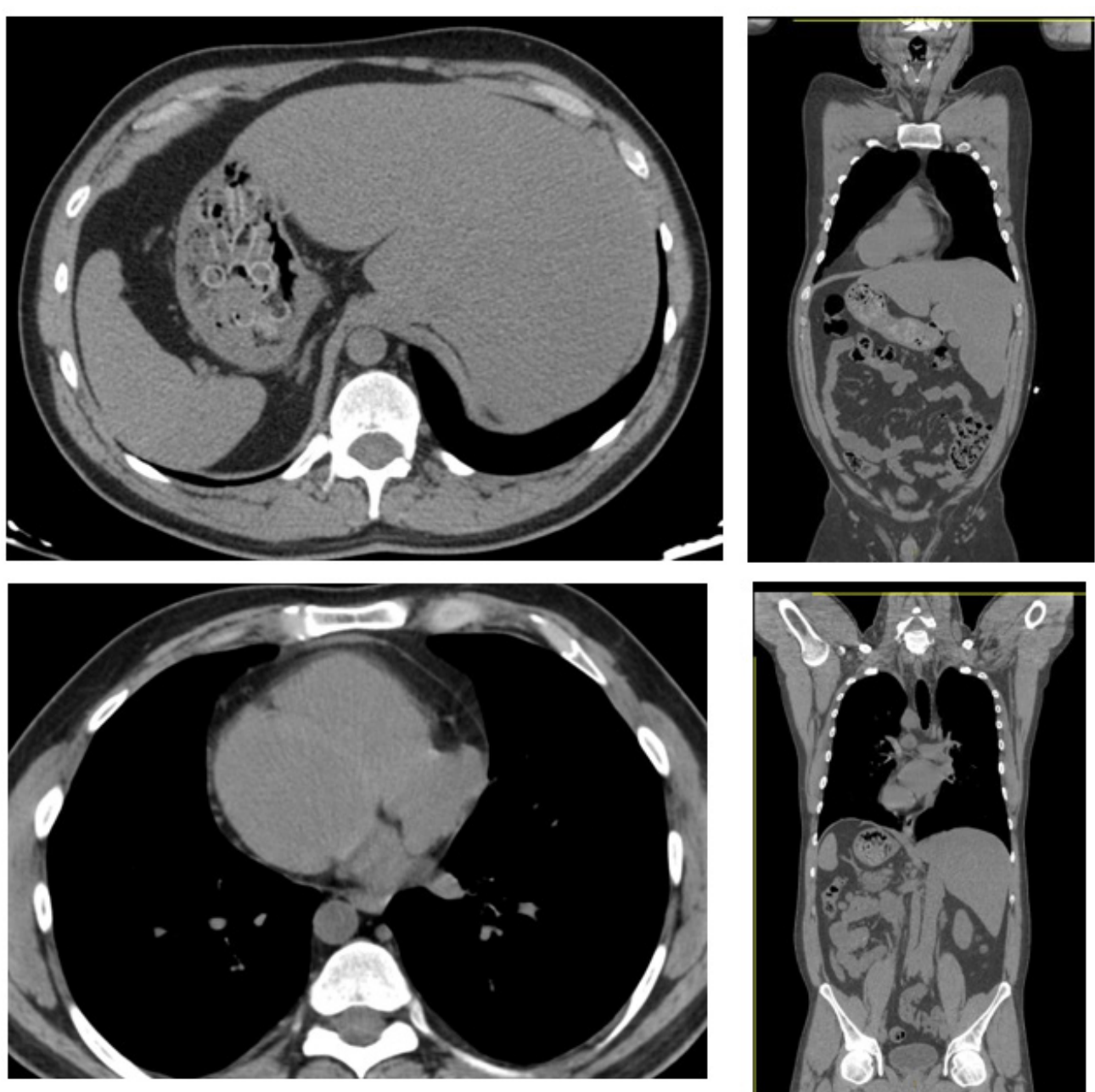

Figure 1: Computed Tomography reconstructions; Axial MPR (on the left) and coronal MPR (on the right) had shown the presence of a situation of situs inversus totalis. Computed Tomography had well-identified a rightrotated cardiac image (dextrocardia), with also an evident rotation of the main vascular structures (thoracic aorta and pulmonary artery). The right lung was bilobed, and the left lung was trilobed. The liver was located in the left upper quadrant, and the spleen was located in the right upper quadrant. The remaining abdominal structures demonstrated a mirror-image location relative to situs solitus. The abdominal aorta was right-sided, the inferior vena cava was left-sided, the pancreatic head and uncinate process were to the left of midline, and the splenic and superior mesenteric artery and vein had a mirror-image positioning.

To determine situs, one may employ chest radiography, ultrasonography, computed tomography, or magnetic resonance imaging to evaluate the following crucial structures: the position of the cardiac apex, position of the atria, position of the aorta relative to mid-line, presence of trilobed or bilobed lungs, position of the stomach, position of the liver and gallbladder, presence and appearance of the spleen(s), and infra-diaphragmatic positioning of the venous drainage relative to mid-line [6]. Chest radiography allows for assessment of situs. When situs is inconclusive, echocardiography will help clarify the cardiovascular anatomy, and real-time abdominal sonography will show the presence or absence of splenic tissue and further define the relationships of the inferior vena cava and abdominal aorta. Computed tomography and magnetic resonance imaging may be necessary when anatomic questions remain $[7,8]$.

\section{Conclusion}

Situs inversus totalis is a rare recessive autosomal congenital abnormality, in which there is a complete inversion of the thoracic and abdominal organs, which constitute a mirror image of the normal anatomy. Computed Tomography, thanks to its reconstructions, can well individuate the spectrum of situs anomalies, that is very important for the diagnosis of disease and setting up possible therapeutic strategies for the patients.

\section{Acknowledgement}

None.

\section{Conflicts of interest}

The authors certify that there is no conflict of interest with any financial organization regarding the material discussed in the manuscript. 


\section{Patient Consent Statement}

The patient had confirmed his consense for the publication of our case report.

\section{References}

1. Fulcher A, Turner MA (2002) Abdominal manifestations of situs anomalies in adults. Radiographics 22(6): 1439-1456.

2. Tonkin IL, Tonkin AK (1982) Visceroatrial situs abnormalities: sonographic and computed tomographic appearance. AJR Am J Roentgenol 138(3): 509-515.

3. Hernanz Schulman M, Ambrosino MM, Genieser NB, D Friedman, H Banner, et al. (1990) Current evaluation of the patient with abnormal visceroatrial situs. AJR Am J Roentgenol 154(4): 797-802.
4. Maldjian PD, Saric M (2007) Approach to dextrocardia in adults: review. AJR Am J Roentgenol 188(6 Suppl): S39-S49.

5. Goo HW, Park IS, Ko JK, Yong Hwue Kim, Dong-Man Seo, et al. (2003) CT of congenital heart disease: normal anatomy and typical pathologic conditions. Radiographics 23 Spec No: S147 - S165.

6. Applegate KE, Goske MJ, Pierce G, Murphy D (1999) Situs revisited: imaging of the heterotaxy syndrome. Radiographics 19(4): 837-852.

7. Choe YH, Kim YM, Han BK, Park KG, Lee HJ (1997) MR imaging in the morphologic diagnosis of congenital heart disease. Radiographics 17(2): 403-422.

8. Paul J Shogan, Col Les Folio (2011) Situs Inversus Totalis. Mil Med 176(7): 840-843. 